\title{
HIDRODINÂMICA E NUTRIENTES INORGÂNICOS DISSOLVIDOS NO ESTUÁRIO DO RIO PEREQUÊ, SC
}

\author{
Signorin, M. ${ }^{1 a}$; Pereira Filho, J. ${ }^{1 b}$; Delfim, R. ${ }^{1 c}$, Schettini, C. A. F. ${ }^{2 \star}$ \\ 1 - Centro de Ciências Tecnológicas da Terra e do Mar - Universidade do Vale do Itajaí (CTTMar/ \\ UNIVALI). Rua Uruguai, 458, Itajaí, SC, Brasil. CEP 88302-202. \\ E-mails: (a) morjanasignorin@gmail.com; (b) jurandir@univali.br; (c) delfim@univali.br \\ 2 - Instituto de Ciências do Mar - Universidade Federal do Ceará (LABOMAR-UFC), Av. da Abolição \\ 3207, Fortaleza, CE, Brasil. CEP 60165-081, \\ *Corresponding author: guto.schettini@gmail.com
}

\begin{abstract}
Signorin, M.; Pereira Filho, J.; Delfim, R., Schettini, C. A. F. 2010. Hydrodynamics and Dissolved Inorganic Nutrients in the Perequê River Estuary, SC. Braz. J. Aquat. Sci. Technol. 14(2): 13-21. ISSN 1808-7035. The estuary of the Perequê River has small dimensions, being the main fluvial input in the Porto Belo Bight and has never been investigated regarding its oceanographic features. A field campaign was carried out where physical and chemical variables were recorded in a fixed sampling station during a complete tide cycle: water level, currents, salinity, temperature, turbity, $\mathrm{pH}$, dissolved oxygen (DO), dissolved inorganic nutrients (ammonium - $\mathrm{NH}_{4}^{+}$, nitrite - $\mathrm{NO}_{2}^{-}$, nitrate - $\mathrm{NO}_{3}^{-}$, silicate $\mathrm{Si}$, and phosphate $-\mathrm{PO}_{4}^{3-}$ ) and chlorophyll-á. A longitudinal survey was done along the estuary to evaluate the salt intrusion. Results showed that flood tide currents were stronger than the ebb currents, with values of 0,5 and $-0,21 \mathrm{~m} . \mathrm{s}$ 1 , respectively. The salinity ranged between 17 and 29, with small stratification during the low tide and no stratification during the high tide. The time variation of the salinity presented a well defined front system, where the salinity presented low values during the low tide and a steep increase during the flood, where the water becomes with coastal water characteristics. The concentration of dissolved inorganic nitrogen (DIN) reached very high levels, reaching $97,7 \mu \mathrm{mol}$.L 1 , mainly because the contribution of $\mathrm{NH}_{4}{ }^{+}(74 \%)$, indicating presence of domestic sewage. The DIN presented inverse relationship with salinity, meanwhile the chlorophyll showed direct relationship. The silicate and phosphate did not presented relationship with salinity. The water quality in the lower estuary was mainly driven by estuary-shelf exchanges, and presented poor horizontal mixing between estuarine and coastal waters.
\end{abstract}

Keywords: circulation, salt intrusion, water quality, pollution.

\section{INTRODUÇÃO}

Estuários são corpos de água costeiros semifechados com ligação livre com oceano aberto, estendendo-se rio acima até o limite da influência da maré, sendo que em seu interior a água do mar é mensuravelmente diluída pela água doce oriunda da drenagem continental (CAMERON \& PRITCHARD, 1963; FAIRBRIDGE, 1980; DYER, 1997), incluindo a plataforma continental adjacente onde a pluma estuarina interage com as águas costeiras mais salinas (MIRANDA, et al., 2002). Um estuário pode ser subdividido em três zonas distintas: a) Zona de maré do rio - parte fluvial com salinidade praticamente igual a zero, ainda sujeita a influência da maré; b) Zona de mistura - região onde ocorre a mistura da água doce da drenagem continental com a água do mar; c) Zona Costeira - região costeira adjacente que se estende até a frente da pluma estuarina, que delimita a camada limite costeira (Kjerfve, 1987). A zona de mistura é onde ocorrem os fortes gradientes longitudinais e verticais da salinidade, onde a intrusão salina no canal é diretamente relacionada com a descarga fluvial e com a maré (Kostaschuk \& Atwood, 1989). A salinidade também pode variar verticalmente, o que distingue estuários quanto à estrutura vertical de salinidade, podendo ser classificados como altamente estratificado, parcialmente estratificado e verticalmente homogêneo (Cameron \& Pritchard, 1963).

Estuários são ambientes de grande importância sócio-econômica e biológica, onde ocorrem intensas transformações de matéria orgânica, devido à abundância e diversidade de sua comunidade e a renovação periódica de sua água, representando um importante ponto de ligação entre os ecossistemas fluviais e marinhos (Pereira Filho et al., 2003). Entretanto, relativamente poucos estuários ao redor do mundo são efetivamente conhecidos ao ponto de que este conhecimento forneça bases para tomadas de decisão para o desenvolvimento sustentável (Schettini, 2002). Os sistemas estuarinos são os principais fornecedores de nutrientes para a região costeira, pois recebem e concentram o material originado de sua bacia de drenagem e podem vir a receber aportes significativos por ação antrópica. Todo esse aporte de nutrientes, matéria prima para a produção primária, coloca os estuários entre os sistemas mais produtivos do mundo, com altas taxas de produção primária e teores de biomassa autótrofa 
e heterótrofa (Pereira Filho et al., 2001). Estima-se que $28 \%$ da produção primária global ocorra em áreas costeiras, sendo que estes sistemas cobrem apenas $8 \%$ da superfície da terra (Jonge et al., 2002). As atividades humanas afetam o balanço de nutrientes, aumentando $\mathrm{o}$ aporte conforme $\mathrm{o}$ adensamento populacional dessas áreas (Nixon, 1992).

Ao longo da costa de Santa Catarina ocorrem dezenas de estuários de pequenas dimensões. Isto se deve a geologia local com a proximidade de altos relevos junto da costa produzindo diversas bacias hidrográficas de pequenas dimensões. Devido às qualidades cênicas do litoral catarinense, este tem sido ocupado ao longo de sua orla de maneira crescente por balneários e loteamentos, que em muitos casos se dá com pouco ou nenhum planejamento. Uma conseqüência é que a ocupação produz efeitos deletérios sobre os cursos de água, sendo estes efeitos potencialmente maiores sobre os cursos de água menores. E, sendo pequenos, recebem pouca ou nenhuma atenção para avaliar o seu papel no ciclo biogeoquímico e contribuição de nutrientes ou poluentes para a zona costeira.

Dadas as suas dimensões reduzidas e proximidade com Itajaí, o estuário do rio Perequê foi utilizado para atividades práticas de coleta e análise de dados físicos e químicos relacionados com as disciplinas de Oceanografia Física Descritiva e Oceanografia Química do Curso de Oceanografia da UNIVALI. A atividade de coleta acompanhou um ciclo completo de maré semidiurna, de 13 horas, o que torna os resultados representativos para uma avaliação inicial das condições oceanográficas do estuário do Rio Perequê. O objetivo deste manuscrito é apresentar estes resultados como uma primeira caracterização das condições físicas e químicas, tendo como motivação a esparsa bibliografia que trata de sistemas de pequenas dimensões (e.g., Schettini et al., 2000; Pereira Filho et al., 2001).

\section{ÁREA DE ESTUDO}

O estuário do rio Perequê está localizado no limite entre os municípios de Porto Belo e Itapema (Figura 1 ; $\left.27^{\circ} 08^{\prime} 41^{\prime \prime} \mathrm{S} \mathrm{e} 48^{\circ} 35^{\prime} 07^{\prime \prime} \mathrm{W}\right)$, Santa Catarina, aproximadamente $55 \mathrm{~km}$ ao norte de Florianópolis. $\mathrm{O}$ estuário apresenta importância local na pesca artesanal e recreativa, e navegação de pequeno porte, sendo a única fonte local de exportação de material continental à porção sul da enseada de Itapema. O estuário apresenta um canal meandrante em uma planície costeira, e ao longo de suas margens existem áreas remanescentes de vegetação de manguezal. As margens no trecho de cerca de $500 \mathrm{~m}$ mais próximos da desembocadura são urbanizados com residências e marinas. A largura do canal aumenta gradativamente no sentido de jusante, sendo da ordem de $50 \mathrm{~m}$, com uma brusca diminuição na desembocadura para $25 \mathrm{~m}$. Na costa adjacente à desembocadura ocorrem formação de bancos de areia associados com o delta de vazante. Durante o período que foi feito este estudo a desembocadura estava sendo dragada para aumento do calado para possibilitar a navegação de embarcações recreativas.

O clima regional é mesotérmico úmido, com superávit hídrico ao longo de todo o ano. Os índices de precipitação e evapotranspiração potencial anuais são da ordem de 1440 e 984 mm.ano-1, respectivamente (GAPLAN, 1986). As chuvas são uniformemente distribuídas ao longo do ano, não havendo uma distinção climatológica de períodos secos e úmidos. Ambos podem ocorrer em qualquer época do ano, ou nem ocorrer.

As marés regionais são do tipo micro-marés mistas com predominância semi-diurna, com altura variando entre 0,4 e 1,2 m entre períodos de quadratura e sizígia, respectivamente (Schettini, 2002). Efeitos meterológicos sobre o nível da água costeiro são importantes, e eventos extremos podem produzir sobreelevações da ordem de $1 \mathrm{~m}$ em relação à maré astronômica (Truccolo et al., 2006). As características físicas da água na plataforma interna de Santa Catarina são principalmente determinadas pela mistura entre as massas de água Água Tropical, Água Central do Atlântico Sul e Água Costeira (e.g. Miranda, 1982; Castro Fo. \& Miranda, 1998), sendo que próximo da costa ocorre predomínio de águas menos salinas influenciadas pelo aporte fluvial local ou pela presença da Pluma do Prata durante o inverno (Schettini et al., 2005; Hille et al., 2008).

\section{MATERIAIS E MÉTODOS}

Uma campanha oceanográfica foi realizada no dia 11 de agosto de 2007 para monitorar, durante um ciclo completo de maré semi-diurna (13 horas), variáveis físicas e químicas no estuário do Rio Perequê (Figura 1). Durante a campanha foram coletadas informações in situ de nível da água, temperatura, salinidade, velocidade e direção de correntes, turbidez, $\mathrm{pH}$ e oxigênio dissolvido. Amostras de água foram coletadas para posterior análise da concentração de nutrientes inorgânicos dissolvidos e clorofila- $\alpha$ em laboratório. Os dados e amostras foram coletados em uma estação situada a 450 m à montante da desembocadura, a partir de uma ponte, e no talvegue da seção transversal. Devido a pouca profundidade do canal, 2,5 $\mathrm{m}$, foram realizadas medições e coleta de água apenas nos ní- 


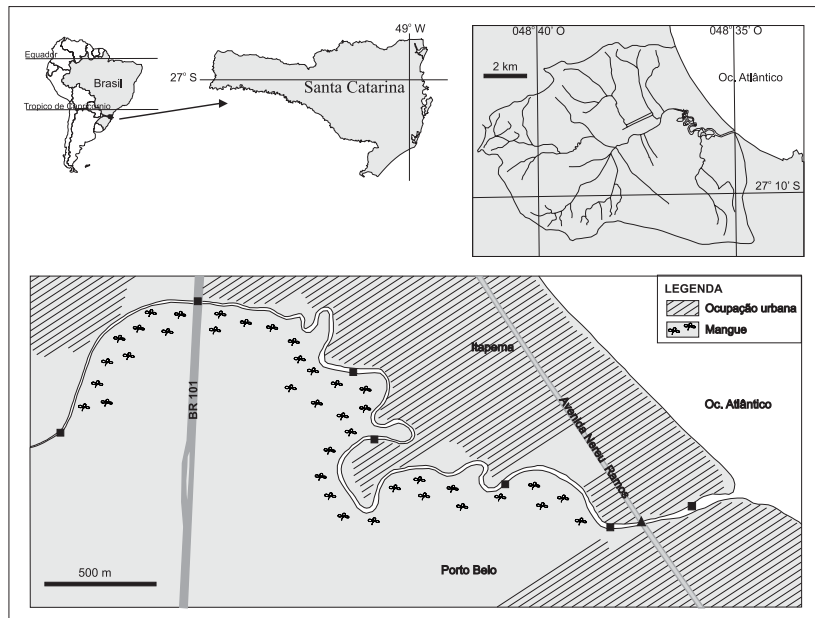

Figura 1 - Localização do estuário do Rio Perequê, indicando a estação de monitoramento temporal (triângulo), e estações de coleta de sal ao longo do estuário (quadrados).

veis de superfície e próximo ao fundo, em intervalos horários.

O nível da água foi monitorado a partir da medição da altura da superfície da água e uma referência fixa, em intervalos de 30 minutos. Dados de velocidade e direção de corrente foram coletados com um correntógrafo Valeport SK-110, que forneceu também dados de temperatura e salinidade. No mesmo ponto foram coletados dados de turbidez (NTU), pH e oxigênio dissolvido (mg.l-1), com uma sonda multi-parâmetro YSI 6600, também nos níveis de superfície e fundo. A sonda foi calibrada com solução padrão antes do início da campanha. Além da amostragem temporal na estação fixa foi realizado um perfil longitudinal do estuário para verificar a distribuição de salinidade e temperatura, durante a preamar. Para isso foi utilizada uma sonda tipo CTD marca Saiv A/S modelo SD204. Estações foram realizadas a cada quilômetro, até $5,5 \mathrm{~km}$ a montante da barra do estuário.

Os dados de velocidade foram reduzidos para componentes de enchente (positivos) e vazante (negativos), a partir da análise da distribuição de freqüência de direção. Os dados foram fortemente bi-modais, e a definição de enchente ou vazante foi a partir dos setores complementares. Os dados de nível de água foram referenciados ao nível médio do período de observação, dada a falta de uma referência de nível.

As amostras de água foram coletadas utilizando de garrafa de Van Dorn. As amostras de água após a coleta foram imediatamente filtradas com filtros Whatman GFF e congeladas. Posteriormente foram determinados os nutrientes $\left(\mathrm{NH}_{4}^{+}, \mathrm{NO}_{2}, \mathrm{NO}_{3}\right.$, Si e $\mathrm{PO}_{4}{ }^{3-}$ ) através de métodos colorimétricos clássicos adaptados de Strickland \& Parsons (1972) descritos em Baumgarten et al. (1996). O total de nitrogenados dissolvidos (NID) foi obtido através da soma das concen- trações de $\mathrm{NH}_{4}^{+}, \mathrm{NO}_{2}^{-}$e $\mathrm{NO}_{3}^{-}$. O método utilizado para quantificação da clorofila- $\alpha$ foi fluorimetria sobre a clorofila- $\alpha$ extraída em acetona $90 \%$, descrito em Parsons \& Maita (1984), utilizando-se de um fluorímetro Turner Design $\AA$ TD-700.

O transporte instantâneo ou transporte residual dos nutrientes e da clorofila- $\alpha$ foi calculado através do

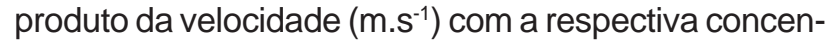
tração $\left(\mathrm{kg} \cdot \mathrm{m}^{-3}\right)$, resultando em transporte de massa $\mathrm{kg} \cdot \mathrm{m}^{-2} \cdot \mathrm{s}^{-1}$. Considerando a pouca variação vertical observada em termos de velocidade e concentrações, foram utilizados valores médios entre superfície e fundo. O transporte total por ciclo de maré $Q$ foi calculado através da integração do transporte residual pelo tempo total da campanha,

$$
Q=\int_{13 h s}\left[\frac{1}{N} \sum_{i=1}^{N} \overline{(u c)_{i}}\right] d t
$$

onde Né o número de amostras temporais, ué a velocidade e cé a concentração de qualquer das variáveis escalares, e a barra indica média vertical. O resultado é dado em $\mathrm{kg} . \mathrm{m}^{-2}$ por ciclo de maré.

Tendo em vista complementar a caracterização do estuário, foi feita uma estimativa da descarga fluvial média do rio Perequê aplicando a equação hidrológica. A vazão do rio Perequê, em $\mathrm{m}^{3} \cdot \mathrm{s}^{-1}$, foi estimada a partir do balanço hidrológico entre precipitação $P$ e evapotranspiração potencial $E p$, ambos em $\mathrm{m} \mathrm{s}^{-1}$, integrados para a área da bacia de drenagem, de acordo com a equação:

$$
V=\int_{\text {Area }}(P-E p) d A
$$

A área da bacia de drenagem do estuário foi obtida em uma carta altimétrica topográfica 1:50.000 do IBGE. Após sua digitalização, a imagem foi georeferenciada no programa Surfer e as latitudes e longitudes dos contornos da bacia foram armazenadas para o posterior cálculo da área da bacia de drenagem, no software MatLab. Foram utilizados dados climatológicos médios das estações de Itajaí, Camboriú e Florianópolis, disponíveis em GAPLAN (1986).

\section{RESULTADOS}

A área da bacia de drenagem do rio Perequê é de $65 \mathrm{~km}^{2}$ (Figura 1) e aplicando a equação hidrológica (Eq. 2), a descarga fluvial climatológica é da ordem de $1,2 \mathrm{~m}^{3} \cdot \mathrm{s}^{-1}$. Aplicando alternativamente a regionalização de vazão a partir de dados medidos na bacia hidrográfica 

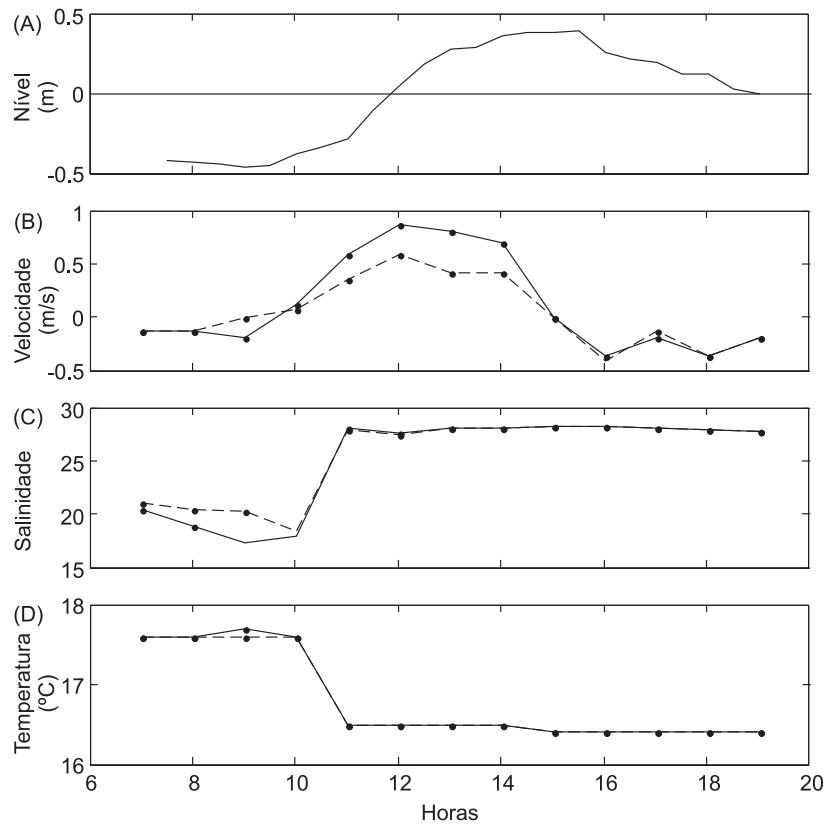

Figura 2 - Variação temporal de (A) nível de água (m), (B) velocidade de correntes $\left(\mathrm{m}^{-1} \mathrm{~s}^{-1}\right),(\mathrm{C})$ salinidade e (D) temperatura $\left({ }^{\circ} \mathrm{C}\right)$. Linha contínua: superfície; linha tracejada: fundo.

do rio Itajaí-Açu apresentado em Schettini (2002), com uma aproximação linear para a área, o resultado foi de $1,3 \mathrm{~m}^{3} \cdot \mathrm{s}^{-1}$, corroborando o primeiro valor.

A previsão da maré astronômica para o dia da campanha fornecida pela Diretoria de Hidrografia e Navegação (DHN) apresentou valores para o Porto de Itajaí de $1,1 \mathrm{~m}$ e $0,2 \mathrm{~m}$ na preamar e baixamar, respectivamente, o que resulta em altura de maré de $0,9 \mathrm{~m}$. A variação do nível medido em campo apresentou valores variando entre o nível médio de 0,4 $\mathrm{m}$ na preamar e $0,46 \mathrm{~m}$ na baixamar, resultando em uma altura de 0,86 m (Fig. 2A). A baixamar foi registrada às 9:00 horas, $e$ a preamar foi registrada às 15:00 horas. O nível da água ao final da campanha foi maior do que o observado no início, ficando próximo do nível médio do período. Os períodos de enchente e vazante não foram iguais. O período de enchente durou cerca de quatro horas (das 10 às 14 horas), e começou a vazar após as 14 horas até o final da campanha (Figura 2B). A velocidade média das correntes de enchente apresentou maior magnitude que a média da velocidade de vazante, 0,50 e $0,21 \mathrm{~m} \cdot \mathrm{s}^{-1}$, respectivamente, e a velocidade residual foi de 0,08 m.s. $^{-1}$ (Tabela 1 ).

O estuário apresentou pequena estratificação de salinidade de poucas unidades no período de maré vazante, até as 10 horas da manhã (Figura 2C), com valores médios de 18,8 e 20,2 para a superfície e fundo, respectivamente. Com a entrada da maré no sistema, houve uma brusca variação da salinidade, aumentando de 20 para 29 em um intervalo de uma hora. Este aumento brusco foi associado com a passagem de uma frente de densidade. A frente foi observada visualmente
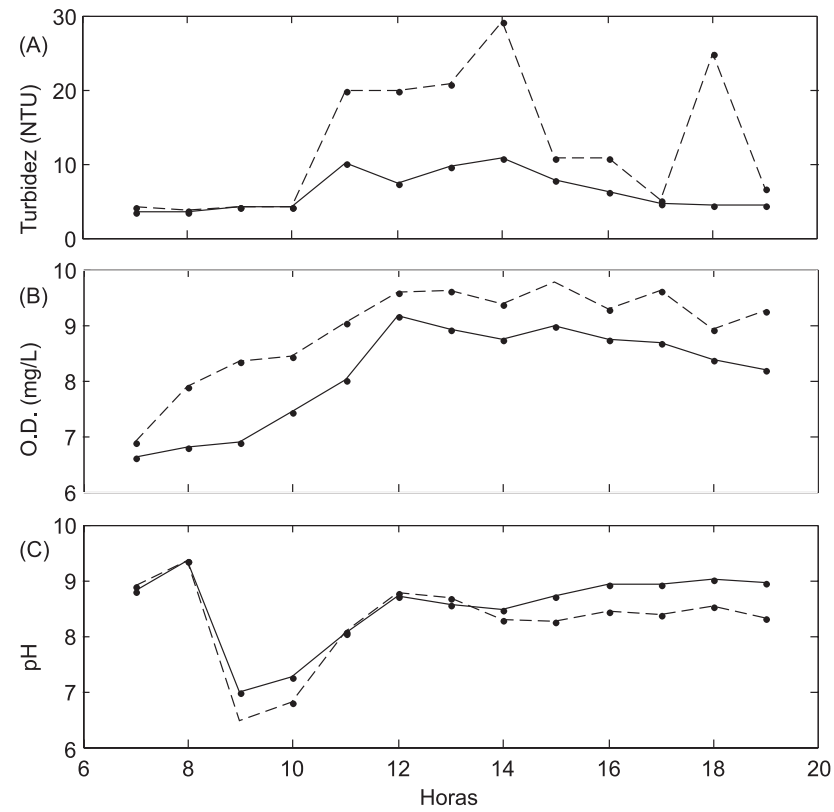

Figura 3 - Variação temporal de (A) turbidez (NTU), (B) oxigênio dissolvido $\left(\mathrm{mg}^{\mathrm{I}} \mathrm{I}^{-1}\right)$, e $(\mathrm{C}) \mathrm{pH}$. Linha contínua: superfície; linha tracejada: fundo.

através de uma linha de espuma separando águas de cores distintas. Após esta variação a salinidade manteve-se constante tanto na superfície quanto no fundo, não apresentando estratificação, até o final da campanha. A temperatura da água apresentou variação temporal de forma inversa à salinidade (Figura 2D). Com a entrada da água marinha no sistema a temperatura diminuiu de $17,6^{\circ} \mathrm{C}$ para $16,4^{\circ} \mathrm{C}$.

A turbidez acompanhou a variação da maré (Figura $3 A$ ). Durante a enchente, às 10 horas, houve um pequeno aumento na superfície, permanecendo praticamente constante até o final da campanha. No fundo a turbidez apresentou maior variação, flutuando conforme a variação da velocidade das correntes. O oxigênio dissolvido (OD) aumentou, tanto na superfície como no fundo, com a entrada da água marinha no sistema (Figura 3B), com média na coluna de água de $6,76 \mathrm{mg} . \mathrm{L}^{-}$ ${ }^{1}$ no início da campanha e $8,73 \mathrm{mg} \cdot \mathrm{L}^{-1}$ no final. O pH também acompanhou a variação da maré (Figura 3C). Nas primeiras horas da campanha houve um decréscimo de aproximadamente duas unidades, passando de 9,3 para 7,0, e com a entrada da maré no sistema houve novo aumento, porém não chegando ao valor inicial, permanecendo praticamente constante na superfície e no fundo com média de 8,5 .

O silicato apresentou maiores concentrações durante a baixamar, sem variação entre superfície e fundo, com valor de médio de $46 \mu \mathrm{mol} . \mathrm{L}^{-1}$ até as 10 horas. Após a inversão da maré a concentração começou a apresentar grandes flutuações, não seguindo nenhum padrão específico. O valor máximo de $56,9 \mu \mathrm{mol} . \mathrm{L}^{-1}$ foi registrado às 9 horas, e valor mínimo de $7,9 \mu \mathrm{mol} . \mathrm{L}^{-1}$, 

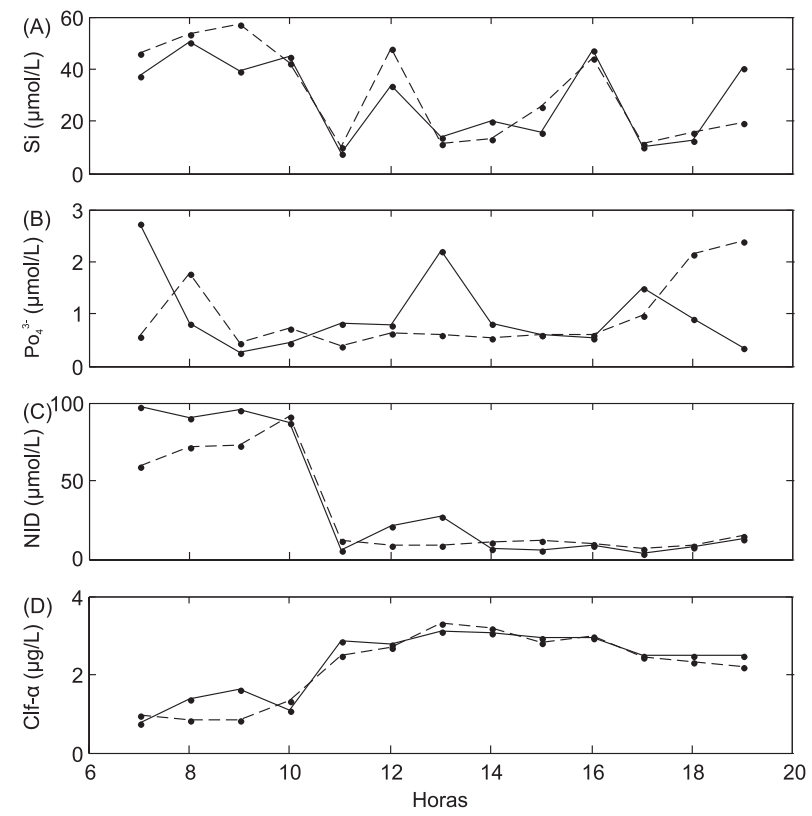

Figura 4 - Variação temporal de (A) silício, (B) fosfato, (C) NID $\left(\mathrm{NH}_{4}{ }^{+}+\mathrm{NO}_{2}{ }^{-}+\mathrm{NO}_{3}\right.$; (D) Clorofila- $\alpha$. Os pontos representam amostras coletadas em superfície e os círculos representam amostras coletadas no fundo.

às 11 horas, logo após o início da entrada de água marinha no sistema (Figura 4A). O fosfato não apresentou relação aparente com a maré (Figura 4B), apresentando valor médio de $0,97 \mu \mathrm{mol} . \mathrm{L}^{-1}$, com valor mínimo de $0,26 \mu$ mol. $L^{-1}$ registrado às 9 horas e máximo de $2,73 \mu \mathrm{mol} . \mathrm{L}^{-1}$, registrado às 7 horas (Tabela 1 ).

Os nutrientes nitrogenados inorgânicos dissolvidos (NID) acompanharam de forma inversa a variação da maré (Figuras 2C e 4C). As maiores concentrações foram registradas no período de vazante no início da campanha, até às 10 horas da manhã. Após a inversão da maré ocorreu uma brusca queda, permanecendo praticamente constante em $9,8 \mu \mathrm{mol} . \mathrm{L}^{-1}$ até o final da campanha. O valor máximo encontrado para o NID foi de $97,7 \mu \mathrm{mol} . \mathrm{L}^{-1}$ às 7 horas da manhã, e o mínimo foi $2,16 \mu \mathrm{mol} . \mathrm{L}^{-1}$, registrado às 17 horas. Em termos gerais, a forma $\mathrm{NH}_{4}{ }^{+}$é predominante, compondo $84 \%$ do $\mathrm{NID}$, seguido pelo $\mathrm{NO}_{3}$ que representa $14 \%$ e pelo $\mathrm{NO}_{2}$ com $2 \%$. A clorofila- $\alpha$ apresentou valores relativamente baixos, variando de 0,76 a 3,34 $\mu \mathrm{g} \cdot \mathrm{L}^{-1}$ (Tabela 1), com um pequeno aumento após a entrada da água marinha no sistema (Figura 4D), e a concentração média em torno de $2,25 \mu \mathrm{g} \cdot \mathrm{L}^{-1}$.

A Figura 5 apresenta o transporte horário de NID, fosfato, silicato e clorofila- $\alpha$, e a Tabela 2 apresenta os valores de transporte residuais e o transporte total integrado para o ciclo de maré. Todas as variáveis acima indicadas apresentaram forte relação com a maré, e todas apresentaram transporte residual estuário adentro. Contudo, distinguindo-se as diferentes formas do NID, o nitrito e nitrato apresentaram transporte residual estuário afora.
Tabela 1 - Média, desvio padrão, valores mínimos e máximos de velocidade de correntes, salinidade, temperatura, turbidez, O.D., $\mathrm{pH}$, nutrientes e clorofila-á durante um ciclo completo de maré, no estuário do rio Perequê, SC.

\begin{tabular}{lcccc}
\hline \hline & Média & $\begin{array}{c}\text { Desvio } \\
\text { Padrão }\end{array}$ & Mínimo & Máximo \\
\hline Veloc. Correntes $\left({\left.\mathrm{m} \cdot \mathrm{s}^{-1}\right)}^{0,08}\right.$ & 0,39 & 0 & $+0,88$ \\
Salinidade & 25,44 & 4,14 & 17,4 & 28,3 \\
Temperatura $\left({ }^{\circ} \mathrm{C}\right)$ & 16,8 & 0,55 & 16,4 & 17,7 \\
Turbidez $(\mathrm{NTU})$ & 9,45 & 7,30 & 3,60 & 29,10 \\
$\mathrm{pH}$ & 8,39 & 0,73 & 6,50 & 9,36 \\
$\mathrm{OD}(\mathrm{mg} / \mathrm{l})$ & 8,5 & 0,94 & 6,63 & 9,77 \\
$\mathrm{Si}(\mu \mathrm{g} / \mathrm{l})$ & 29,70 & 16,51 & 7,88 & 56,97 \\
$\mathrm{PO}_{4}{ }^{-}(\mu \mathrm{mol} / \mathrm{l})$ & 0,97 & 0,70 & 0,26 & 2,73 \\
$\mathrm{NO}_{2}{ }^{-}(\mu \mathrm{mol} / \mathrm{l})$ & 0,46 & 0,70 & 0,01 & 2,28 \\
$\mathrm{NO}_{3}{ }^{-}(\mu \mathrm{mol} / \mathrm{l})$ & 4,68 & 3,73 & 0,69 & 13,74 \\
$\mathrm{NH}_{4}{ }^{+}(\mu \mathrm{mol} / \mathrm{l})$ & 27,18 & 31,96 & 0,19 & 87,46 \\
$\mathrm{NID}(\mu \mathrm{mol} / \mathrm{l})$ & 32,33 & 35,56 & 2,16 & 97,72 \\
$\mathrm{Clorofila- \alpha}(\mu \mathrm{g} / \mathrm{l})$ & 2,25 & 0,84 & 0,76 & 3,34 \\
\hline \hline
\end{tabular}

A distribuição longitudinal de salinidade apresentou diminuição linear para montante (Figura 6), com valores maiores de salinidade no fundo e tendência de aumento do grau de estratificação para montante. Até a distância de 5,6 quilômetros da desembocadura do sistema a salinidade registrada foi de 1,3 e 13,1 para superfície e fundo, respectivamente. Aplicando-se uma aproximação linear, estima-se que a salinidade estende-se até 9,5 quilômetros à montante.

\section{DISCUSSÃO}

O estuário do rio Perquê apresenta dimensões reduzidas, sendo um ambiente raso. A partir da profundidade alcançada pelo CTD durante os levantamentos de salinidade ao longo do estuário, estima-se que a média da profundidade seja da ordem de 1,5 m. Considerando a escala de largura de $25 \mathrm{~m}$ e extensão de 15 $\mathrm{km}$, seu volume fica da ordem de $5,6 \times 10^{5} \mathrm{~m}^{3}$. O prisma de maré durante a campanha (e.g., Miranda et al., 2002), calculado pelo produto entre a área da superfície livre pela altura da maré, é de $3,0 \times 10^{5} \mathrm{~m}^{3}$, ou aproximadamente a metade do volume do estuário. Estes valores sugerem uma alta taxa de renovação com a plataforma interna. Contudo, o que foi observado a partir dos dados físicos e químicos é que somente um volume restrito a porção mais inferior do estuário é regularmente trocada, e o grau de mistura interna entre a água estuarina e a água costeira é limitada.

O cenário acima descrito pode ser considerado com uma aproximação para o comportamento do estuário na maior parte do ano, dada as características climatológicas regionais. É esperado que na maior parte do tempo a vazão do rio Perquê seja baixa, aumentando somente durante períodos de precipitação, similarmente ao que ocorre em outros estuários próximos 

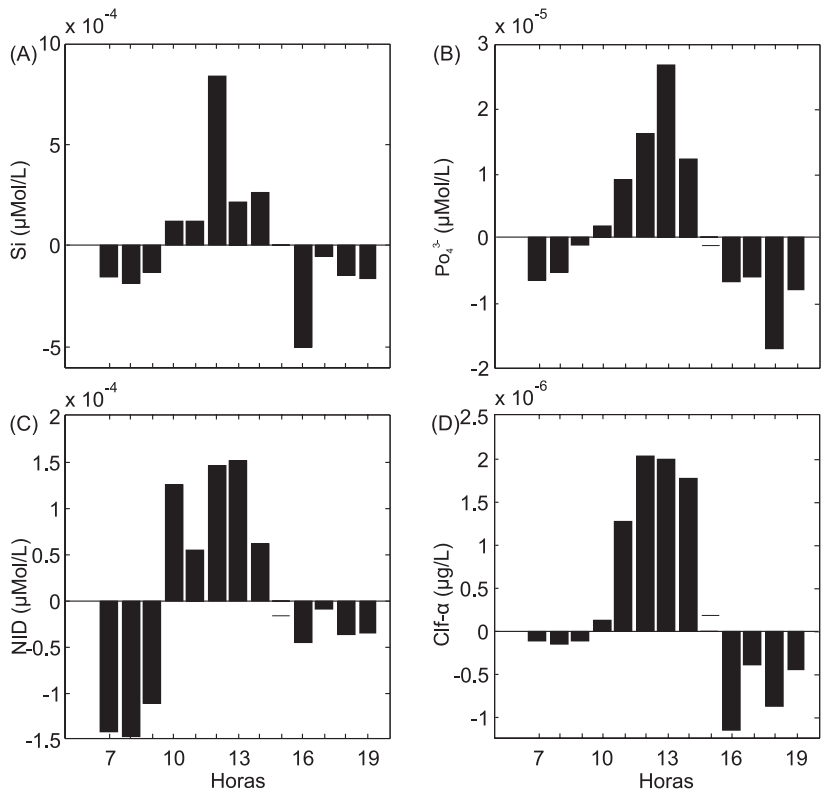

Figura 5 - Transporte residual do (A) Silicato; (B) Fosfato; (C) NID; (D) Clorofila- $\alpha$ ao longo do ciclo de maré em $\mathrm{kg} \cdot \mathrm{m}^{-2} \cdot \mathrm{s}^{-1}$.

(Schettini, 2002). A região não apresenta uma distinção entre períodos chuvosos e secos, sendo as chuvas uniformemente distribuídas ao longo do ano (Gaplan, 1986). Durante os períodos chuvosos possivelmente haverá uma reflexo das característica em função da evolução da onda de cheia, o que irá despejar água doce diretamente sobre a plataforma adjacente. Mas estes períodos apresentam duração curta da ordem de horas a poucos dias (Schettini, 2002).

O grau de interação entre águas estuarina e costeira é indicado pela variação temporal da salinidade e temperatura (Figura 2C e D), e pelas relações entre o NID e a clorofila- $\alpha$ com a salinidade (Figura $7 C$ e D), que não são tão evidentes para o fosfato e silicato (Figuras 7A e B). O NID foi dominado pela presença de amônio, que por sua vez é um indicador de contaminação orgânica doméstica (Pereira Filho et al., 2001). A clorofila- $\alpha$, por outro lado, apresenta valores maiores nas águas costeiras e mais salinas adjacente ao estuário (e.g. Pereira Filho et al., 2001; Schettini et al., 2005). Ainda que os níveis de nutrientes no estuário sejam elevados, a turbidez também o é, fazendo com

Tabela 2 - Transporte residual e transporte total dos nutrientes e da clorofila- $\alpha$ durante um ciclo completo de maré no estuário do rio Perequê, SC.

\begin{tabular}{lcc}
\hline \hline & $\begin{array}{c}\text { Transporte Residual } \\
\mathbf{k g} \cdot \mathbf{s}^{-1} \cdot \mathbf{m}^{-2}\left(\mathbf{x} \mathbf{1 0}^{-5}\right)\end{array}$ & $\begin{array}{c}\text { Transporte Total } \\
\mathbf{k g} \cdot \mathbf{m}^{-2}\end{array}$ \\
\hline $\mathrm{Si}$ & 1,86 & 0.87 \\
$\mathrm{P}-\mathrm{PO}_{4}{ }^{3-}$ & 0,11 & 0.05 \\
$\mathrm{NID}$ & 0,07 & 0.03 \\
$\mathrm{~N}-\mathrm{NO}_{3}{ }^{-}$ & $-0,07$ & -0.03 \\
$\mathrm{~N}-\mathrm{NO}_{2}{ }^{-}$ & $-0,03$ & -0.02 \\
$\mathrm{~N}-\mathrm{NH}_{4}{ }^{+}$ & 0,18 & 0.08 \\
Clorofila- $\alpha$ & 0,03 & 0.01 \\
\hline \hline
\end{tabular}

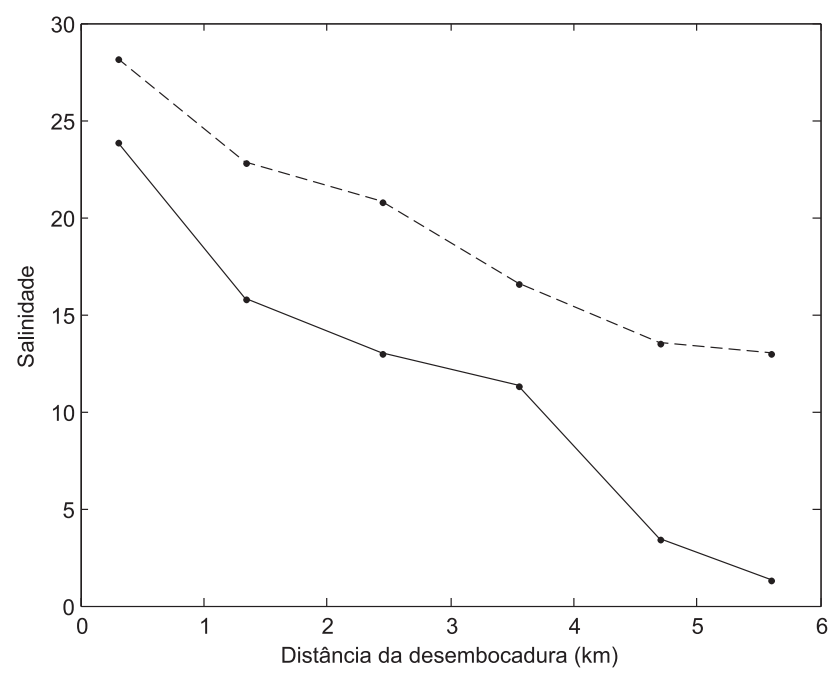

Figura 6 - Variação espacial da salinidade, onde pontos representam salinidade de superfície e os círculos representam salinidade de fundo.

que a luminosidade seja um fator limitante para a produção primária. Os nutrientes apresentaram maiores concentrações na vazante, associados com a água estuarina e maior influência da drenagem continental. As elevadas concentrações de $\mathrm{NH}_{4}{ }^{+}$no sistema evidenciam uma contaminação do ambiente por esgoto doméstico, devido à intensa ocupação urbana das margens. Outros autores também encontraram elevada concentração de $\mathrm{NH}_{4}{ }^{+}$(e.g. Pereira Filho et al., 2002; Pereira Filho et al., 2003; Schettini et al., 2000), associadas da mesma forma à atividades antrópicas, tais como lançamento de efluentes domésticos.
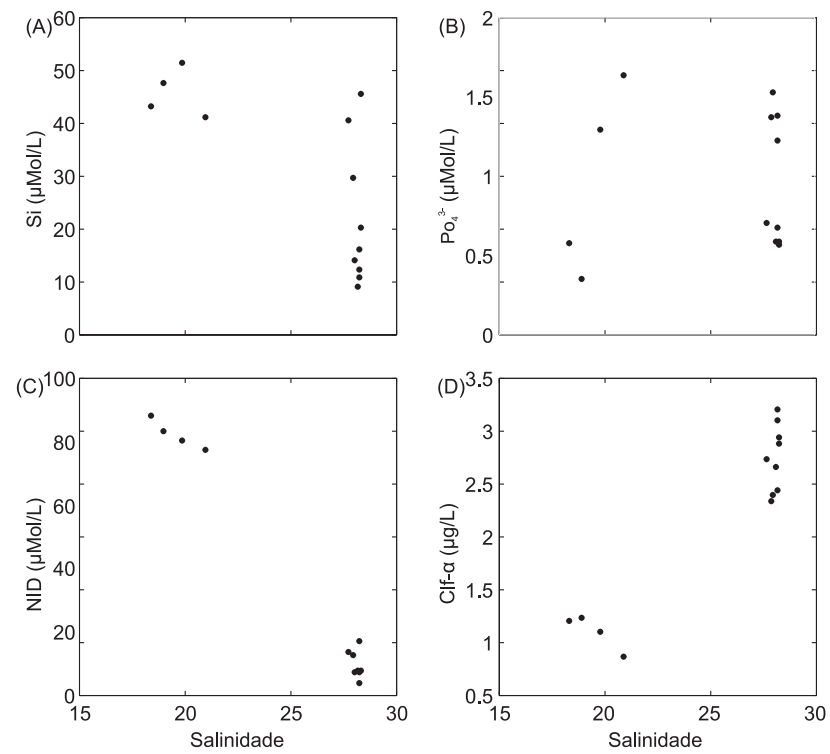

Figura 7 - Correlação da salinidade com: (A) Silício; (B) Fosfato; (C) NID; (D) Clorofila- $\alpha$. 

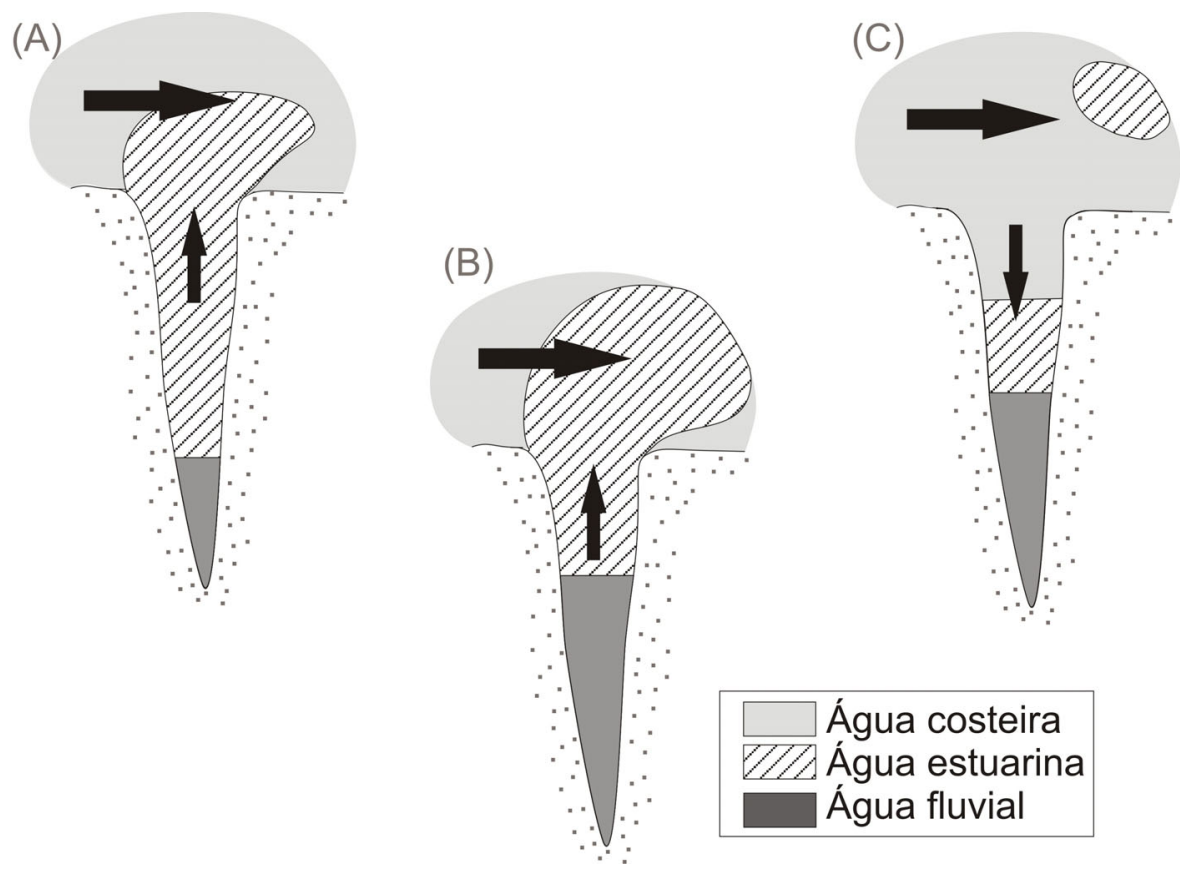

Água costeira

$\mathbb{Z}$ Água estuarina

Água fluvial

Figura 8 - Modelo esquemático da circulação do estuário do Rio Perequê e da sua relação com a plataforma adjacente.

A circulação do estuário produz um padrão de distribuição de salinidade verticalmente homogêneo próximo da desembocadura. A condição homogênea da salinidade durante várias horas foi observada após a passagem da frente de densidade estuário adentro. Antes da passagem da frente a coluna de água apresentava alguma estratificação, o que também foi registrado ao longo do estuário. Isto sugere que as velocidades de corrente e a pequena profundidade do estuário não produzem uma mistura vertical eficiente nas águas mais interiores, e também não produz uma mistura longitudinal com a água costeira que entre durante a enchente.

A Figura 8 apresenta um modelo conceitual do sistema de troca de água observado no estuário do rio Perequê. Durante a maré vazante, o volume de água do estuário é advectado para a plataforma adjacente; correntes costeiras induzem o deslocamento deste volume ao longo da costa; com a maré enchente, um volume equivalente de água costeira advecta estuário adentro. No ciclo de maré seguinte, a maior parte do volume que entrou será renovado, e apenas uma pequena fração da água estuarina será trocada. Pereira Filho et al. (2001) apresentou um modelo similar para o estuário do rio Camboriú, tendo como indicadores a clorofila- $\alpha$ e o carbono orgânico particulado. Comparativamente, o estuário do rio Camboriú apresenta dimensões maiores do que o estuário do rio Perequê, embora ainda seja um estuário pequeno ( $\sim 50 \mathrm{~m}$ de largura, $2 \mathrm{~m}$ de profundidade e $10 \mathrm{~km}$ de extensão) e fortemente impactado pelo desenvolvimento urbano.
Os transportes residuais dos nutrientes inorgânicos dissolvidos e de clorofila- $\alpha$ foram para montante. Isto se deve a desigualdade semi-diurna da maré (e.g. Pugh, 1987), pois o nível ao final da campanha foi maior do que no início, ainda que completando um ciclo de maré de treze horas. A assimetria na variação das marés enchente e vazante, com velocidades mais intensas para a enchente do que para a vazante, resultou na permanência de um volume de água marinha após o ciclo monitorado, e consequentemente dos constituintes dissolvidos.

Ainda ocorrem nas margens do estuário do rio Perequê vegetação de manguezal. Embora a área de cobertura não seja muito extensa em termos absolutos (por inspeção visual sobre imagem de satélite), ele é em termos de escala do estuário. Embora o presente estudo não permita fazer uma avaliação do papel do manguezal sobre os fluxos de nutrientes, é possível que estes sejam importantes (e.g. Dittmar \& Lara, 2001; Rezende et al., 1990 \& 2007), e devam ser investigados futuramente.

\section{CONSIDERAÇÕES FINAIS}

O estuário do rio Perequê é um estuário de dimensões pequenas, similarmente a dezenas de outros sistemas ao longo da costa de Santa Catarina. O regime de circulação observado foi fortemente influenciado pela maré, porém a mistura entre a água estuarina e costeira é limitada. Isto produz um regime de frente de 
densidade que desloca-se estuário adentro durante o período de enchente de maré. As águas estuarinas apresentaram elevadas concentrações de amônio, o que pode ser atribuído à poluição doméstica. O estuário apresentou transporte de nutrientes para o interior durante o período amostrado. Contudo, este balanço deve ser considerado como preliminar. No caso deste sistema, somente uma campanha de 25 horas poderá fornecer uma balanço de água que indicará o efetivo transporte de escalares.

\section{AGRADECIMENTOS}

Agradecemos ao Professor Luíz Bruner de Miranda do Instituto Oceanográfico da Universidade de São Paulo que gentilmente cedeu o correntógrafo Valeport utilizado na campanha. Agradecemos também à Marina Porto do Rio, localizada no município do Porto Belo, SC, por ceder o espaço para realizarmos o trabalho no dia da campanha. Morjana Signorin é bolsista ProUni. Bolsa CNPq-306217/2007-4.

\section{REFERÊNCIAS}

Baumgarten, M.G.Z. 1996. Manual de análises em oceanografia química. Editora da FURG, Rio Grande, $132 p$.

Cameron, W.M. \& Pritchard D.W. 1963. Estuaries. In: Hill, M.N. (ed.) The Sea. Vol. 2. John Wiley and Sons, New York. 306-324pp.

Castro, B.M. \& Miranda, L.B. 1998. Physical oceanography of the Western Atlantic Continental Shelf located between $4^{\circ} \mathrm{N}$ and $34^{\circ} \mathrm{S}$. In: Robinson, A.R. \& Brink, K.H. (eds.) The Sea. Vol. 11. John Wiley \& Sons, New York. 209-251pp.

Dittmar, T. \& Lara, R.J. 2001. Do mangroves rather than rivers provide nutrients to coastal environments south of the Amazon River? Evidence from long-term flux measurements. Marine Ecology Progress Series, 213: 67-77.

Dyer, K.R. 1997. Estuaries: a physical introduction. 2a Edição. John Wiley and Sons, New York, 195p.

Fairbridge, R.W. 1980. The estuary its definition and geodynamic cycle. In: Olausson, E. \& Cato, I. (eds.) Chemistry and Biogeochemistry of Estuaries. Wiley, New York. 1-35pp.

Gaplan - Gabinete de Planejamento de Santa Catarina. 1986. Atlas de Santa Catarina. Aerofoto Cruzeiro, Rio de Janeiro, 173p.

Hille, E.; Schettini, C.A.F.; Ribeiro, M.R. 2008. Estrutura termohalina no litoral de Santa Catarina nos anos de 2005 e 2006. In: Braga, E.S. (ed.) Oceano- grafia e mudanças globais. Edusp, São Paulo. 371$381 p p$.

Jonge, V.N. de; Elliot, M; Orive, E. 2002. Causes, historical development, effects and future challenges of a common environmental problem: eutrophication. Hydrobiologia. 475/476:1-19.

Kjerfve, B. 1990. Manual for investigation of hydrological processes in mangrove ecosystems. Unesco, $\mathrm{Pa}-$ ris, $79 p$.

Kostaschuk, R.A. \& Atwood, L.A. 1989. River discharge and tidal controls on salt-wedge position and implications for channel shoaling: Fraser River, British Columbia. Canadian Journal of Civil Engineering. 17(3):452-459.

Miranda, L.B. 1982. Análise de massas de água da plataforma continental e da região oceânica adjacente: Cabo de São Tomé (RJ) à llha de São Sebastião (SP). Tese de Livre Docência. Instituto Oceanográfico - USP. 194p.

Miranda, L.B.; Castro, B.M.; Kjerfve, B. 2002. Princípios de Oceanografia física de estuários. Edusp, São Paulo, 414p.

Nixon, S.W. 1982. Nutrient Dynamics, Primary Production and Fisheries Yields of Lagoons. Oceanologica Acta. Suppl. 4:357-371.

Parsons, T.R. \& Maita, Y. 1984. A manual of chemical and biological methods for seawater analysis. Pergamon, Oxford, 173p.

Pereira Filho, J.; Schettini, C.A.F.; Rörig, L.; Siegle, E. 2001. Intratidal variation and net Transport of Dissolved Inorganic Nutrients, POC and Chlorophyll a in the Camboriú River Estuary, Brazil. Estuarine, Coastal and Shelf Science. 53:249-257.

Pereira Filho J.; Spillere, L.C.; Schettini, C.A.F.; Silva, L.F. 2002. Estuário do rio Camboriú-SC: variação intramareal e transporte residual de nutrientes, COP e clorofila- $\alpha$ em condições de quadratura e sizígia. Notas Técnicas FACIMAR. 6:137-151.

Pereira Filho J.; Spillere, L.C.; Schettini, C.A.F. 2003. Dinâmica e nutrientes na região portuária do estuário do rio Itajaí-açu, SC. Atlântica. 25(1): 11-20.

Pugh, D.T. 1987. Tides, surges and mean sea level. New York, John Wiley and Sons, 472p.

Rezende, C.E.; Lacerda, L.D.; Ovalle, A.R.C.; Silva, C.A.R.E. \& Martinelli, L.A. 1990.Nature of POC transport in a mangrove ecosystem: A carbon stable isotopic study. Estuarine, Coastal and Shelf Science, 30: 641-646.

Rezende, C.E.; Lacerda, L.D.; Ovalle, A.R.C.; \& Silva, L.F.F. 2007. Dial organic carbon fluctuations in a mangrove tidal creek in Sepetiba Bay, Southeast Brazil. Brazilian Journal of Biology, 67: 673-680.

Schettini, C.A.F. 2002. Caracterização física do estuário do rio Itajaí-Açu. Revista Brasileira de Recursos Hídricos. 7(1):123-142. 
Schettini, C.A.F; Pereira Filho, J.; Spillere, L. 2000. Caracterização oceanográfica e biogeoquímica dos estuários dos rios Tavares e Defuntos, Reserva Extrativista de Pirajubaé, Florianópolis, SC. Notas Técnicas FACIMAR. 4:11-28.

Schettini, C.A.F.; Resgalla Jr., C.; Pereira Filho, J.; Silva, M.A.C.; Truccolo, E.C.; Rörig, L.R. 2005. Variabilidade temporal das características oceanográficas e ecológicas da região de influência fluvial do
Rio Itajaí-açu. Brazilian Journal of Aquatic Science and Technology. 9(2):93-102.

Truccolo, E.C.; Franco, D.; Schettini, C.A.F. 2006. The low frequency sea level oscillations in the northern coast of Santa Catarina. Brazilian Journal of Coastal Research. 39(SI):547-552.

Submetido: Dezembro/2008 Revisado: Abril/2009 Aceito: Outubro/2010 